\title{
On Surface-Plasmon-Polariton Waves Excited in the Turbadar-Otto Configuration
}

\author{
Tom G. Mackay ${ }^{1,2}$ (D) Muhammad Faryad ${ }^{3}$
}

Received: 18 June 2021 / Accepted: 5 November 2021 / Published online: 15 December 2021

(c) The Author(s) 2021

\begin{abstract}
A local minimum in the plot of linear reflectance versus angle of incidence, on its own, is insufficient to identify a surfaceplasmon-polariton wave (SPPW). Further checks are required in order to confirm the identity of a SPPW. The wavenumber should be compared with that extracted from the dispersion relation for the corresponding canonical boundary-value problem. Also, for prism-coupled configurations such as the Turbadar-Otto configuration which are based on SPPW-excitation via evanescent waves, the angle of incidence should be greater than the critical angle needed for total internal reflection.
\end{abstract}

Keywords Surface-plasmon polariton wave $\cdot$ Dispersion relation $\cdot$ Evanescent wave

\section{Introduction}

Surface-plasmon-polariton waves (SPPWs) are guided by the planar interface of a dielectric material and a metal [1]. These surface waves cannot be excited by direct illumination. Instead, indirect methods of excitation are required, such as prism-coupled methods that utilize evanescent waves to excite SPPWs [2]. An example of such a method is provided by the Turbadar-Otto configuration [3, 4], as schematically illustrated in Fig. 1. This configuration was recently used to investigate SPPWs at the planar interface of a columnar thin film (CTF) made from $\mathrm{TiO}_{2}$ and a thin film of $\mathrm{Ag}$ [5].

The authors of Ref. [5] used local minimums in plots of linear reflectance versus angle of incidence (labeled $\theta$ in Fig. 1) to identify SPPWs. However, this means of identifying SPPWs is not sufficiently discriminating: a local

Tom G. Mackay

T.Mackay@ed.ac.uk

1 School of Mathematics and Maxwell Institute for Mathematical Sciences, University of Edinburgh, Edinburgh EH9 3JZ, UK

2 NanoMM-Nanoengineered Metamaterials Group, Department of Engineering Science and Mechanics, Pennsylvania State University, University Park, PA 16802 6812, USA

3 Department of Physics, Lahore University of Management Sciences, Lahore, Pakistan minimum is consistent with the excitation of a SPPW but there may well be other explanations for local minimums, such as cavity resonances [6, 7]. It is essential that wavenumbers are compared with those extracted from the dispersion relation for the corresponding canonical boundaryvalue problem in order to confirm the identity of suspected SPPWs [8].

Using plots of linear reflectance versus angle of incidence in their Fig. 10 (corresponding to CTF vapor deposition angle $\chi_{v}=5^{\circ}$ ), the authors of Ref. [5] claim to identify two SPPWs: one for the angle of incidence $\theta=46^{\circ}$ and the other for the angle of incidence $\theta=62^{\circ}$. The local minimum at $\theta=46^{\circ}$ is attributed to the Ag-air interface while the local minimum at $\theta=62^{\circ}$ is attributed to the CTF-Ag interface. Since the thickness of the Ag film is $40 \mathrm{~nm}$ and its skin depth is $23.6 \mathrm{~nm}$ (at the chosen free-space wavelength of $633 \mathrm{~nm}$ ), there should be very little interaction between surface waves at the Ag-air interface and the CTF-Ag interface. On the basis of wavenumbers extracted from the dispersion relation for the corresponding canonical boundary value problems, angles of incidence in the neighborhood of $\theta=42.92^{\circ}$ and $\theta=62.05^{\circ}$ for the Ag-air interface and the CTF-Ag interface, respectively, are compatible with SPPW excitation. Thus, in the cases of the two claimed SPPWs in Fig. 10 of Ref. [5], the angles of incidence derived from the canonical boundary-value problems are broadly in agreement with those inferred from the plots of linear reflectance.

Let us now turn to plots of linear reflectance versus angle of incidence in Fig. 11 (corresponding to CTF vapor 


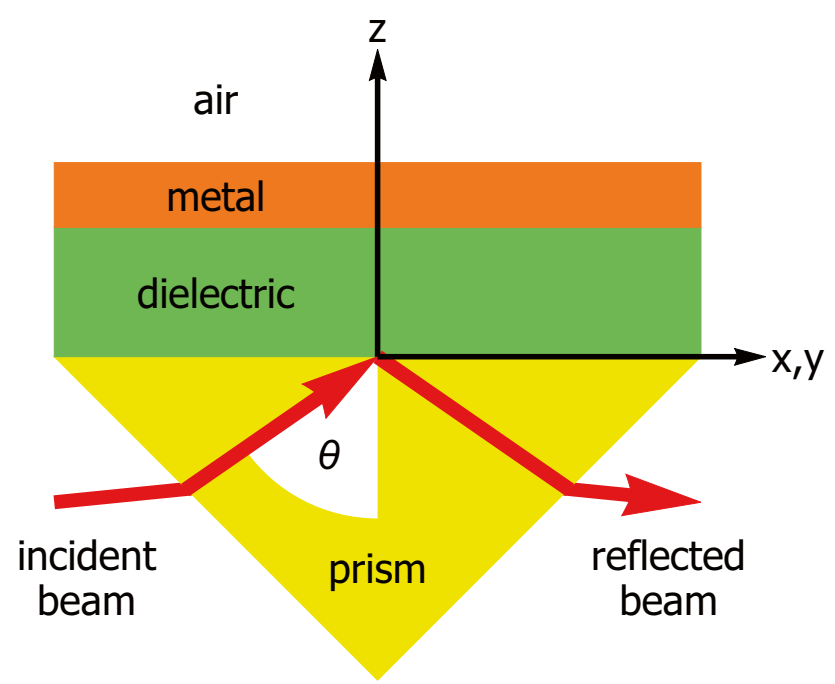

Fig. 1 Schematic illustration of the Turbadar-Otto configuration

deposition angle $\chi_{v}=15^{\circ}$ ) of Ref. [5]. The authors claim to identify two SPPWs: one for the angle of incidence $\theta=31^{\circ}$ and the other for the angle of incidence $\theta=70^{\circ}$. The local minimum in the linear reflectance plot at $\theta=31^{\circ}$ is attributed to the Ag-air interface while the local minimum in the linear reflectance plot at $\theta=70^{\circ}$ is attributed to the CTF-Ag interface. On the basis of wavenumbers extracted from the dispersion relation for the corresponding canonical boundary value problems, angles of incidence in the neighborhood of $\theta=42.92^{\circ}$ and $\theta=67.76^{\circ}$ for the Ag-air interface and the CTF-Ag interface, respectively, are compatible with SPPW excitation. The angle of incidence derived from the canonical boundary-value problem for the CTF-Ag interface (i.e., $\theta=67.76^{\circ}$ ) is broadly in agreement with that inferred from the plot of linear reflectance in Fig. 11 of Ref. [5] (i.e., $\theta=70^{\circ}$ ). However, the same cannot be said for the Ag-air interface: the angle of incidence derived from the canonical boundary-value problem for the Ag-air interface differs by $12^{\circ}$ from the angle of incidence for the corresponding local minimum in the plot of linear reflectance. Therefore, the local minimum in the linear reflectance plot at $\theta=31^{\circ}$ in Fig. 11 cannot be the signature of a SPPW.

Furthermore, in order to excite a SPPW at the Ag-air interface for the Turbadar-Otto configuration, light entering the air region from the region occupied by the Ag thin film must be in the form of an evanescent wave [1,2]. Therefore, the angle of incidence in the prism must be greater than the critical angle for total internal reflection at the planar interface of the prism and air. This critical angle is $\sin ^{-1}(1 / n)=41.46^{\circ}$, where $n=1.5105$ is the refractive index of the prism in Ref. [5]. Consequently, it is not possible to excite a SPPW wave at an angle of incidence $\theta=31^{\circ}$, contrary to the claim made in Ref. [5].
In closing, a local minimum in the plot of linear reflectance versus angle of incidence, on its own, is insufficient to identify a SPPW. Additional checks are needed to confirm the identity of a SPPW: The wavenumber should be compared with that extracted from the dispersion relation for the corresponding canonical boundary-value problem. And, for prism-coupled configurations which are based on SPPW-excitation via evanescent waves, the angle of incidence should be greater than the critical angle needed for total internal reflection. Failure to carry out these additional checks has led to a mistakenly identified SPPW in Ref. [5].

Author Contributions Both authors worked on the concept; TGM wrote the first draft; MF helped to finalize.

Funding This work was supported in part by EPSRC (grant number $\mathrm{EP} / \mathrm{S} 00033 \mathrm{X} / 1)$.

\section{Declarations}

Ethics Approval Not applicable.

Consent to Participate Not applicable.

Consent for Publication Both authors consent to publication.

Conflict of Interest The authors declare no competing interests.

Open Access This article is licensed under a Creative Commons Attribution 4.0 International License, which permits use, sharing, adaptation, distribution and reproduction in any medium or format, as long as you give appropriate credit to the original author(s) and the source, provide a link to the Creative Commons licence, and indicate if changes were made. The images or other third party material in this article are included in the article's Creative Commons licence, unless indicated otherwise in a credit line to the material. If material is not included in the article's Creative Commons licence and your intended use is not permitted by statutory regulation or exceeds the permitted use, you will need to obtain permission directly from the copyright holder. To view a copy of this licence, visit http://creativecommons.org/licenses/by/4.0/.

\section{References}

1. Pitarke JM, Silkin VM, Chulkov EV, Echenique PM (2007) Theory of surface plasmon and surface-plasmon polaritons. Rep Prog Phys 70:1-87

2. Polo JA Jr, Mackay TG, Lakhtakia A (2013) Electromagnetic surface waves: a modern perspective. Elsevier, Waltham, MA, USA

3. Turbadar T (1964) Complete absorption of plane polarized light by thin metal films. Opt Acta 11:207-210

4. Otto A (1968) Excitation of nonradiative surface plasma waves in silver by the method of frustrated total reflection. Z Phys 216:398-410

5. Babaei F, Seyyedi SA (2021) Excitation of surface plasmonpolariton wave at both interfaces of a silver thin film in two-layer Kretschmann geometry. Plasmonics. https://doi.org/10.1007/ s11468-021-01476-9 
6. Mackay TG (2019) On the identification of surface waves in numerical studies. Plasmonics 14:1-2

7. Faryad M, Polo JA Jr, Lakhtakia A (2010) Multiple trains of samecolor surface plasmon-polaritons guided by the planar interface of a metal and a sculptured nematic thin film. Part IV: Canonical problem. J Nanophoton 4:043505
8. Polo JA Jr, Lakhtakia A (2008) Morphological effects on surfaceplasmon-polariton waves at the planar interface of a metal and a columnar thin film. Opt Commun 281:5453-5457

Publisher's Note Springer Nature remains neutral with regard to jurisdictional claims in published maps and institutional affiliations. 